\title{
MEASURES ON EFFECT ALGEBRAS
}

\author{
LUO LAIZHEN ${ }^{凶}$ and LI RONGLU
}

\author{
(Received 15 August 2007)
}

Abstract

In this paper, by introducing the bounded variation measure defined on effect algebras, we present the equivalent conditions about uniformly strongly additive measures.

2000 Mathematics subject classification: 81P03.

Keywords and phrases: effect algebras, strongly additive, vector-valued measures.

\section{Preliminaries}

Foulis and Bennett in 1994 introduced the algebraic system $(L, \oplus, 0,1)$ to model unsharp quantum logics, and $(L, \oplus, 0,1)$ is said to be an effect algebra [1].

Let $L$ be a set with two special elements 0,1 , and let $\perp$ be a subset of $L \times L$. If $(a, b) \in \perp$, write $a \perp b$. Let $\oplus: \perp \rightarrow L$ be a binary operation. Suppose that the following axioms hold.

(E1) If $a, b \in L$ and $a \perp b$, then $b \perp a$ and $a \oplus b=b \oplus a$ (commutative law).

(E2) If $a, b, c \in L, a \perp b$ and $(a \oplus b) \perp c$, then we have $b \perp c, a \perp(b \oplus c)$ and $(a \oplus b) \oplus c=a \oplus(b \oplus c)$ (associative law).

(E3) For every $a \in L$ there exists a unique $b \in L$ such that $a \perp b$ and $a \oplus b=1$ (orthosupplementation law).

(E4) If $a \in L$ and $1 \perp a$, then $a=0$ (zero-one law).

Then $(L, \oplus, 0,1)$ is called an effect algebra.

If $a, b \in L$ and $a \perp b$, we say that $a$ and $b$ are othogonal. If $a \oplus b=1$ we say that $b$ is the orthosupplementation of $a$, and we write $b=a^{\prime}$. Clearly $1^{\prime}=0,\left(a^{\prime}\right)^{\prime}=a, a \perp 0$ and $a \oplus 0=a$ for all $a \in L$. We say that $a \leq b$ if there exists $c \in L$ such that $a \perp c$ and $a \oplus c=b$. We know that $\leq$ is a partial order on $L$ and satisfies the conditions that $0 \leq a \leq 1, a \leq b \Leftrightarrow b^{\prime} \leq a^{\prime}$ and $a \leq b^{\prime} \Leftrightarrow a \perp b$ for $a, b \in L$.

If $a \leq b$, the element $c \in L$ such that $c \perp a$ and $c \oplus a=b$ is unique, and satisfies the condition $c=\left(a \oplus b^{\prime}\right)^{\prime}$. It will be denoted $c=b \ominus a$.

(c) 2008 Australian Mathematical Society 0004-9727/08 \$A2.00+0.00 
Let $F=\left\{a_{i}: 1 \leq i \leq n\right\}$ be a finite subset of $L$. If $a_{1} \perp a_{2},\left(a_{1} \oplus a_{2}\right) \perp a_{3}, \ldots$ and $\left(a_{1} \oplus a_{2} \oplus \cdots \oplus a_{n-1}\right) \perp a_{n}$, we say that $F$ is orthogonal and we define

$$
\bigoplus F=a_{1} \oplus a_{2} \oplus \cdots \oplus a_{n-1} \oplus a_{n}=\left(a_{1} \oplus a_{2} \oplus \cdots \oplus a_{n-1}\right) \oplus a_{n} .
$$

Now, if $A$ is an arbitrary subset of $L$ and $\mathcal{F}(A)$ is the family of all finite subsets of $A$, we say that $A$ is orthogonal if $F$ is orthogonal for every $F \in \mathcal{F}(A)$. If $A$ is orthogonal, we define

$$
\bigoplus A=\bigvee\{\bigoplus F: F \in \mathcal{F}(A)\}
$$

Moreover, let $\left(a_{i}\right)_{i \in I}$ be an orthogonal subset of $L$. Then we know that [3]:

(1) if $I$ is finite and $J \subseteq I$, then

$$
\left(\bigoplus_{i \in J} a_{i}\right) \perp\left(\bigoplus_{i \in I \backslash J} a_{i}\right)
$$

and

$$
\bigoplus_{i \in I} a_{i}=\left(\bigoplus_{i \in J} a_{i}\right) \oplus\left(\bigoplus_{i \in I \backslash J} a_{i}\right)
$$

(2) if $J \subseteq I$ and there exist $a=\bigoplus_{i \in I} a_{i}, b=\bigoplus_{i \in J} a_{i}$ and $c=\bigoplus_{i \in I \backslash J} a_{i}$, then $b \perp c$ and $a=b \oplus c$;

(3) if there exists $\bigoplus_{i \in M} a_{i}$ for all $M \subseteq I$ and $\left\{H_{j}: j \in J\right\}$ is a partition of $I$, then $A=\left\{\bigoplus_{i \in H_{j}} a_{i}: j \in J\right\}$ is an orthogonal subset of $L$, there exists $\oplus A$ and $\oplus A=\bigoplus_{i \in I} a_{i}$;

(4) if $\left(F_{j}\right)_{j \in J}$ is a family of finite and pairwise disjoint subsets of $I$, then the set $\left\{\bigoplus_{i \in F_{j}} a_{i}: j \in J\right\}$ is an orthogonal subset of $L$;

(5) if $b_{i} \in L$ and $b_{i} \leq a_{i}$ for $i \in I$, then $\left(b_{i}\right)_{i \in I}$ is an orthogonal subset of $L$.

In what follows, let $L$ be an effect algebra, $X$ be a Banach space and $\mu: L \rightarrow X$ be a vector measure. The variation of $\mu$ is the nonnegative function $|\mu|$ whose value on an element $a \in L$ is given by

$$
|\mu|(a)=\sup _{\Delta} \sum_{a_{j} \in \Delta}\left\|\mu\left(a_{j}\right)\right\|,
$$

where $\Delta=\left\{a_{1}, a_{2}, \ldots, a_{n}\right\}$ such that $a_{1} \oplus a_{2} \oplus \cdots \oplus a_{n}=a, a_{j} \in L$ for all $j=$ $1,2, \ldots, n$.

If $|\mu|(1)<\infty$, we call $\mu$ a measure of bounded variation.

The semivariation of $\mu$ is the nonnegative function $\|\mu\|$ whose value on an element $a \in L$ is defined by

$$
\|\mu\|(a)=\sup \left\{\left|x^{*} \mu\right|(a): x^{*} \in X^{*},\left\|x^{*}\right\| \leq 1\right\},
$$

where $\left|x^{*} \mu\right|$ is the variation of the real-valued measure $x^{*} \mu$. 
If $\|\mu\|(1)<\infty$, we call $\mu$ a measure of bounded semivariation.

$\mu: L \rightarrow X$ is said to be strongly additive if, for any orthogonal sequence $\left(a_{n}\right)$ of $L$, the series $\sum_{n=1}^{\infty} \mu\left(a_{n}\right)$ converges in $X$.

A family of strongly additive vector measures $\left\{\mu_{\tau}: \tau \in T\right\}$ is said to be uniformly strongly additive if, for any orthogonal sequence $\left(a_{n}\right)$ of $L$, the series

$$
\lim _{m}\left\|\sum_{n=m}^{\infty} \mu_{\tau}\left(a_{n}\right)\right\|=0
$$

uniformly in $\tau \in T$.

$\mu: L \rightarrow X$ is said to be bounded if, for any orthogonal sequence $\left(a_{n}\right)$ of $L$, $\left\{\mu\left(a_{n}\right)\right\}_{n=1}^{\infty}$ is bounded.

$\mu: L \rightarrow X$ is said to be strongly bounded if, for any orthogonal sequence $\left(a_{n}\right)$ of $L, \lim _{n \rightarrow \infty} \mu\left(a_{n}\right)=0$.

$\mu: L \rightarrow X$ is said to be countably additive if, for any orthogonal sequence $\left(a_{n}\right)$ of $L$,

$$
\mu\left(\bigoplus_{n=1}^{\infty} a_{n}\right)=\sum_{n=1}^{\infty} \mu\left(a_{n}\right) .
$$

Clearly, a strongly additive vector measure on effect algebras is strongly bounded and a strongly bounded vector measure on effect algebras is bounded.

\section{Main results}

Proposition 1. Let $\mu: L \rightarrow X$ be a vector measure. Then, for $a \in L$,

$$
\|\mu\|(a)=\sup _{\Delta}\left\{\left\|\sum_{j} \varepsilon_{j} \mu\left(a_{j}\right)\right\|\right\},
$$

where $\Delta=\left\{a_{1}, a_{2}, \ldots, a_{n}\right\}$ such that $a_{1} \oplus a_{2} \oplus \cdots \oplus a_{n}=a, a_{j} \in L$ for all $j=1,2, \ldots, n$ and $\left|\varepsilon_{j}\right| \leq 1$.

PROOF. If $a=a_{1} \oplus a_{2} \oplus \cdots \oplus a_{n}, \quad\left\{a_{1}, a_{2}, \ldots, a_{n}\right\}$ is a partition of $a$ into orthogonal members of $L$ and $\varepsilon_{j}$ are scalars such that $\left|\varepsilon_{j}\right| \leq 1$, then

$$
\begin{aligned}
\left\|\sum_{j=1}^{m} \varepsilon_{j} \mu\left(a_{j}\right)\right\| & =\sup \left\{\left|x^{*}\left(\sum_{j=1}^{m} \varepsilon_{j} \mu\left(a_{j}\right)\right)\right|: x^{*} \in X^{*},\left\|x^{*}\right\| \leq 1\right\} \\
& \leq \sup \left\{\sum_{j=1}^{m}\left|\varepsilon_{j} x^{*} \mu\left(a_{j}\right)\right|: x^{*} \in X^{*},\left\|x^{*}\right\| \leq 1\right\} \\
& \leq \sup \left\{\sum_{j=1}^{m}\left|x^{*} \mu\left(a_{j}\right)\right|: x^{*} \in X^{*},\left\|x^{*}\right\| \leq 1\right\} \\
& \leq \sup \left\{\left|x^{*} \mu\right|(a): x^{*} \in X^{*},\left\|x^{*}\right\| \leq 1\right\} \\
& =\|\mu\|(a) .
\end{aligned}
$$


On the other hand, let $x^{*} \in X^{*}$ with $\left\|x^{*}\right\| \leq 1$ and $a=a_{1} \oplus a_{2} \oplus \cdots \oplus a_{n}$, $\left\{a_{1}, a_{2}, \ldots, a_{n}\right\}$ be a partition of $a$ into orthogonal members of $L$. Then

$$
\begin{aligned}
\sum_{j=1}^{m}\left|x^{*} \mu\left(a_{j}\right)\right| & =\sum_{j=1}^{m}\left(\operatorname{sgn} x^{*} \mu\left(a_{j}\right)\right) x^{*} \mu\left(a_{j}\right) \\
& =x^{*}\left(\sum_{j=1}^{m}\left(\operatorname{sgn} x^{*} \mu\left(a_{j}\right)\right) \mu\left(a_{j}\right)\right) \\
& \leq\left\|\sum_{j=1}^{m} \varepsilon_{j} \mu\left(a_{j}\right)\right\| \\
& \leq \sup _{\Delta}\left\|\sum_{j} \varepsilon_{j} \mu\left(a_{j}\right)\right\| .
\end{aligned}
$$

This proves the result.

Proposition 2. Let $\mu: L \rightarrow X$ be a vector measure. Then

$\sup \{\|\mu(h)\|: h \leq e, h \in L\} \leq\|\mu\|(e) \leq 4 \sup \{\|\mu(h)\|: h \leq e, h \in L\}$.

PROOF. For any $e \in L$,

$\sup \{\|\mu(h)\|: h \leq e, h \in L\}=\sup \left\{\sup \left\{\left|x^{*} \mu(h)\right|: x^{*} \in X^{*},\left\|x^{*}\right\| \leq 1\right\}: h \leq e, h \in L\right\}$

$$
\leq\|\mu\|(e) \text {. }
$$

On the other hand, let $x^{*} \in X^{*}$ with $\left\|x^{*}\right\| \leq 1$ and $e=e_{1} \oplus e_{2} \oplus \cdots \oplus e_{n}$, $\left\{e_{1}, e_{2}, \ldots, e_{n}\right\}$ be a partition of $e$ into orthogonal members of $L$. Then

$$
\begin{aligned}
\sum_{i=1}^{n}\left|x^{*} \mu\left(e_{i}\right)\right| & =\sum_{i \in M^{+}} x^{*} \mu\left(e_{i}\right)-\sum_{i \in M^{-}} x^{*} \mu\left(e_{i}\right) \\
& =x^{*}\left(\sum_{i \in M^{+}} \mu\left(e_{i}\right)\right)-x^{*}\left(\sum_{i \in M^{-}} \mu\left(e_{i}\right)\right) \\
& \leq 2 \sup \{\|\mu(h)\|: h \leq e, h \in L\},
\end{aligned}
$$

where

$$
M^{+}=\left\{i: x^{*} \mu\left(e_{i}\right) \geq 0,1 \leq i \leq n\right\} \quad \text { and } \quad M^{-}=\left\{i: x^{*} \mu\left(e_{i}\right)<0,1 \leq i \leq n\right\} .
$$

If $X$ is a complex Banach space, it is easy to see that a similar estimate holds if the number 2 is replaced by the number 4 .

Consequently, a vector measure is of bounded semivariation on $L$ if and only if its range is bounded in $X$.

TheOREM 3. Let $\mu_{\tau}: L \rightarrow X, \tau \in T$, be a family of vector measures. The following statements are equivalent. 
(I) $\left\{\mu_{\tau}: \tau \in T\right\}$ is uniformly strongly additive.

(II) $\left\{x^{*} \mu_{\tau}: \tau \in T, x^{*} \in X^{*},\left\|x^{*}\right\| \leq 1\right\}$ is uniformly strongly additive.

(III) If $\left(a_{n}\right)$ is a sequence of orthogonal members of $L$, then $\lim _{n \rightarrow \infty}\left\|\mu_{\tau}\left(a_{n}\right)\right\|=0$ uniformly in $\tau \in T$.

(IV) If $\left(a_{n}\right)$ is a sequence of orthogonal members of $L$, then $\lim _{n \rightarrow \infty}\left\|\mu_{\tau}\right\|\left(a_{n}\right)=0$ uniformly in $\tau \in T$.

(V) $\left\{\left|x^{*} \mu_{\tau}\right|: \tau \in T, x^{*} \in X^{*},\left\|x^{*}\right\| \leq 1\right\}$ is uniformly strongly additive.

PROOF. (I) $\Rightarrow$ (II), (II) $\Rightarrow$ (III), and (V) $\Rightarrow$ (I) are obvious.

(III) $\Rightarrow$ (IV) If not, there exist a $\delta>0$ and an orthogonal sequence $\left(a_{n}\right)$ of $L$ such that $\sup _{\tau \in T}\left\|\mu_{\tau}\right\|\left(a_{n}\right) \geq 4 \delta>0$ holds for all $n \in N$. By Proposition 1, for every $n$ there is an $h_{n} \in L$ such that $h_{n} \leq a_{n}$ and $\sup _{\tau \in T}\left\|\mu_{\tau}\right\|\left(a_{n}\right) \leq 4 \sup _{\tau \in T}\left\|\mu_{\tau}\left(h_{n}\right)\right\|$. The sequence $\left(h_{n}\right)$ is orthogonal such that

$$
\sup _{\tau \in T}\left\|\mu_{\tau}\left(h_{n}\right)\right\| \geq \delta>0
$$

for every $n \in N$. This shows that (III) implies (IV).

$(\mathrm{IV}) \Rightarrow(\mathrm{V})$. Suppose that $\left\{\left|x^{*} \mu_{\tau}\right|: \tau \in T, x^{*} \in X^{*},\left\|x^{*}\right\| \leq 1\right\}$ is not uniformly strongly additive. Then there exist an orthogonal sequence $\left(a_{n}\right)$ of $L$ and a $\delta>0$ such that, for all $m \in N$,

$$
\sup \left\{\sum_{n=m}^{\infty}\left|x^{*} \mu_{\tau}\right|\left(a_{n}\right): \tau \in T, x^{*} \in X^{*},\left\|x^{*}\right\| \leq 1\right\} \geq 2 \delta>0 .
$$

Thus there is an increasing sequence $\left(m_{j}\right)$ of positive integers such that, for all $j$,

$$
\begin{aligned}
& \sup \left\{\sum_{n=m_{j}+1}^{m_{j+1}}\left|x^{*} \mu_{\tau}\right|\left(a_{n}\right): \tau \in T, x^{*} \in X^{*},\left\|x^{*}\right\| \leq 1\right\} \\
& \quad=\sup \left\{\left|x^{*} \mu_{\tau}\right|\left(\bigoplus_{n=m_{j}+1}^{m_{j+1}} a_{n}\right): \tau \in T, x^{*} \in X^{*},\left\|x^{*}\right\| \leq 1\right\} \geq \delta>0 .
\end{aligned}
$$

Therefore, putting

$$
h_{j}=\bigoplus_{n=m_{j}+1}^{m_{j+1}} a_{n}
$$

$\left(h_{n}\right)$ is an orthogonal sequence of $L$ such that

$$
\sup \left\{\left\|\mu_{\tau}\right\|\left(h_{j}\right): \tau \in T\right\}=\sup \left\{\left|x^{*} \mu_{\tau}\right|\left(h_{j}\right): \tau \in T, x^{*} \in X^{*},\left\|x^{*}\right\| \leq 1\right\} \geq \delta>0 .
$$

This leads to a contradiction. So (V) holds.

Corollary 4. Let $\mu: L \rightarrow X$ be a vector measure. The following statements are equivalent. 
(I) $\quad \mu$ is strongly additive.

(II) $\left\{x^{*} \mu: x^{*} \in X^{*},\left\|x^{*}\right\| \leq 1\right\}$ is uniformly strongly additive.

(III) $\mu$ is strongly bounded, that is, if $\left(a_{n}\right)$ is an orthogonal sequence of members of $L$, then $\lim _{n \rightarrow \infty} \mu\left(a_{n}\right)=0$.

(IV) $\|\mu\|$ is strongly bounded, that is, if $\left(a_{n}\right)$ is an orthogonal sequence of members of $L$, then $\lim _{n \rightarrow \infty}\|\mu\|\left(a_{n}\right)=0$.

(V) $\left\{\left|x^{*} \mu\right|: x^{*} \in X^{*},\left\|x^{*}\right\| \leq 1\right\}$ is uniformly strongly additive.

(VI) $\lim _{n} \mu\left(a_{n}\right)$ exists for every nondecreasing monotone sequence $\left(a_{n}\right)$ of $L$.

(VII) $\lim _{n} \mu\left(a_{n}\right)$ exists for every nonincreasing monotone sequence $\left(a_{n}\right)$ of $L$.

PROOF. The equivalence of (I)-(V) is clear from Theorem 3. And it is also clear that (VI) is equivalent to (VII).

(I) $\Rightarrow$ (VI). Let $\left(a_{n}\right)$ be an orthogonal sequence of $L$ satisfying $a_{1} \leq a_{2} \leq \cdots \leq a_{n}$, and let $c_{n}=a_{n} \ominus a_{n-1}$. Then

$$
\lim _{n} \mu\left(a_{n}\right)=\mu\left(a_{1}\right)+\lim _{n} \sum_{n=2}^{\infty} \mu\left(a_{n} \ominus a_{n-1}\right)
$$

exists since the sequence $\left(c_{n}\right)_{n=2}^{\infty}$ is an orthogonal sequence of $L$.

On the other hand, let $\left(a_{n}\right) \subseteq L$ be an orthogonal sequence, $b_{k}=\bigoplus_{n=1}^{k} a_{n}$ for $k \in N$. Then $\left(b_{k}\right)$ is a nondecreasing sequence of $L$. Then

$$
\lim _{n} \mu\left(a_{n}\right)=\lim _{n}\left[\mu\left(\bigoplus_{n=1}^{k} a_{n}\right)-\mu\left(\bigoplus_{n=1}^{k-1} a_{n}\right)\right]=0 .
$$

This completes the proof.

THEOREM 5. Let $\mu: L \rightarrow X$ be a bounded vector measure. If $L$ satisfies the finite chain condition, that is, no infinite subcollection of $L$ can be orthogonal, then $\mu$ is countably additive.

Proof. Suppose that $L$ satisfies the finite chain condition, and $\left(a_{n}\right)$ is an orthogonal sequence of $L$; then $a_{n}=0$ for all large $n \geq n_{0}, n_{0} \in N$. Hence by finite additivity of $\mu$,

$$
\mu\left(\bigoplus_{n=1}^{\infty} a_{n}\right)=\mu\left(\bigoplus_{n=1}^{n_{0}} a_{n}\right)=\sum_{n=1}^{n_{0}} \mu\left(a_{n}\right)=\sum_{n=1}^{\infty} \mu\left(a_{n}\right),
$$

and $\mu$ is countably additive.

THEOREM 6. If $X$ is a Banach space containing no copy of $c_{0}, \mu: L \rightarrow X$ is a bounded vector measure, then $\mu$ is strongly additive.

PROOF. Since $\mu$ is bounded, for every orthogonal sequence $\left(a_{n}\right)$ of $L$ the series $\left\{\sum_{n=1}^{m} \mu\left(a_{n}\right)\right\}_{m=1}^{\infty}$ is weakly unconditional Cauchy [2], that is, $\sum_{n=1}^{\infty}\left|x^{*} \mu\left(a_{n}\right)\right|<\infty$, for any $x^{*} \in X^{*}$. Therefore, $\left(\mu\left(a_{j}\right)\right)_{j}$ is $c_{0}$-multiplier convergent. Since $X$ contains no copy of $c_{0}$, then $\sum_{n=1}^{\infty} \mu\left(a_{n}\right)$ convergent. Thus $\mu$ is strongly additive. 


\section{References}

[1] D. J. Foulis and M. K. Bennett, 'Effect algebras and unsharp quantum logics', Found. Phys. 24 (1994), 1331-1352.

[2] H. T. Hwang, L. L. Li and H. Kim, 'Bounded vector measures on effect algebras', Bull. Austral. Math. Soc. (2) 72 (2005), 291-298.

[3] F. G. Mazario, 'Convergence theorems for topological group valued measures on effect algebras', Bull. Austral. Math. Soc. 64 (2001), 213-231.

LUO LAIZHEN, Department of Mathematics, Harbin Institute of Technology, Harbin 150006, People's Republic of China

e-mail: luolaizhen@126.com

LI RONGLU, Department of Mathematics, Harbin Institute of Technology, Harbin 150006, People's Republic of China 